\title{
Effect of Heat Treatment on Stress-Induced Martensitic Transformation and Associated Pseudoelasticity in Monocrystalline Cu-Al-Ni Alloys*
}

\author{
By Hidekazu Sakamoto**, Yoshihiro Nakai*** and Ken'ichi Shimizu**
}

\begin{abstract}
Effects of the heat treatment on the characteristics of various types of stress-induced martensitic transformations and associated pseudoelasticity in single crystals with $\langle 001\rangle$ orientation of $\mathrm{Cu}-14.1 \mathrm{Al}-$ 4.2Ni and $\mathrm{Cu}-14.7 \mathrm{Al}-4.2 \mathrm{Ni}$ (mass\%) alloys have been investigated. Three kinds of heat treatments were given, that is, (a) rapid quench from $1273 \mathrm{~K}$, (b) normal quench from $1273 \mathrm{~K}$ and (c) aging at $523 \mathrm{~K}$ for $3.6 \mathrm{ks}$ after the normal quench. As a result, various parameters characterizing the transformations have been found to vary with the heat treatment. $M_{\mathrm{s}}$ temperatures and critical stresses for inducing $\gamma_{1}^{\prime} \rightarrow \beta_{1}^{\prime \prime}$ and $\beta_{1}^{\prime}\left(\beta_{1}^{\prime \prime}\right) \rightarrow \alpha_{1}^{\prime}$ transformations increase in the order of (a), (b) and (c), whereas critical stresses for inducing $\beta_{1} \rightarrow \gamma_{1}^{\prime}$ and $\beta_{1} \rightarrow \beta_{1}^{\prime}$ transformations decrease in that order. These variations indicate that a previously observed anomaly on the temperature dependence of critical stresses for inducing the $\beta_{1}^{\prime} \rightarrow \alpha_{1}^{\prime}$ transformation above $470 \mathrm{~K}$ is due to an aging effect, and further that the interphase boundaries in a phase diagram with the temperature-stress coordinate shift by the heat treatment. Elongations due to various transformations have also been found to vary not only with heat treatment but also with test temperature. This suggests that lattice constants of various martensite phases vary with heat treatment and temperature. The critical stress and the elongation for $\beta_{1}^{\prime}\left(\beta_{1}^{\prime \prime}\right) \rightarrow \alpha_{1}^{\prime}$ transformation have been found to vary in an opposite direction with temperature and heat treatment, suggesting the existence of a critical point for the transformation.
\end{abstract}

(Received September 29, 1986)

\begin{abstract}
Keywords: stress-induced martensitic transformation, transformation pseudoelasticity, copperaluminum-nickel alloy, shape memory alloy, heat treatment, transformation temperature, critical stress, thermal hysteresis, stress hysteresis, transformation elongation
\end{abstract}

\section{Introduction}

Successive stress-induced martensitic transformations and associated pseudoelasticity have so far been extensively studied by using single crystals of $\mathrm{Cu}-\mathrm{Al}-\mathrm{Ni}$ alloys above and below $\mathbf{M}_{\mathrm{s}}{ }^{(1)(2)}$. Three types of two stage pseudoelasticity loops have been observed depending on test temperature $(T)$. The first type, termed type A here, is due to successive $\gamma_{1}^{\prime} \rightarrow \beta_{1}^{\prime \prime}$ and $\beta_{1}^{\prime \prime} \rightarrow \alpha_{1}^{\prime}$ transformations on loading and $\alpha_{1}^{\prime} \rightarrow \beta_{1}^{\prime}$ and $\beta_{1}^{\prime} \rightarrow \gamma_{1}^{\prime}$ transformations on

* A part of this paper was originally read at the Annual Meeting of the Japan Institute of Metals, Hiroshima, (1984).

** The Institute of Scientific and Industrial Research, Osaka University, 8-1, Mihoga-oka, Ibaraki, Osaka 567, Japan.

*** Graduate Student, Osaka University. Present address: R \& D group, Sumitomo Electric Industry Ltd., 1-1-3, Shimaya, Konohana-ku, Osaka 554, Japan. unloading at $T$ below $\mathrm{A}_{\mathrm{f}}$, the second type (type B) due to successive $\beta_{1} \rightarrow \beta_{1}^{\prime}$ and $\beta_{1}^{\prime} \rightarrow \alpha_{1}^{\prime}$ transformations on loading and their successive reversions on unloading at $T$ between $A_{\mathrm{f}}$ and a critical temperature $T_{\mathrm{c}}$, and the third type (type C) due to successive $\beta_{1} \rightarrow \beta_{1}^{\prime}$ and $\beta_{1}^{\prime} \rightarrow \alpha_{1}^{\prime}$ transformations on loading and consecutive $\alpha_{1}^{\prime} \rightarrow \beta_{1}^{\prime} \rightarrow \beta_{1}$ transformations on unloading at $T$ above $T_{\mathrm{c}}$. Here, $\beta_{1}$ is the parent phase with $\mathrm{D}_{3}$ type superlattice and $\gamma_{1}^{\prime}, \beta_{1}^{\prime}, \beta_{1}^{\prime \prime}$ and $\alpha_{1}^{\prime}$ are all martensite phases with close-packed structures having different stacking orders; they are $\gamma_{1}^{\prime}: 2 \mathrm{H}(1 \overline{1}), \quad \beta_{1}^{\prime}: 18 \mathrm{R}(2 \overline{1})_{6}, \quad \beta_{1}^{\prime \prime}: 18 \mathrm{R}(1 \overline{1} 3 \overline{1})_{3} \quad$ and $\alpha_{1}^{\prime}: 6 \mathrm{R}(1)_{6}$. A phase diagram in the temperature-stress coordinates has been determined, by plotting the critical stresses $\left(\sigma_{c}\right)$ for the transformations as a function of $T$. However, an elusive problem remains in a high temperature region of the phase diagram as described below.

Below about $470 \mathrm{~K}, \sigma_{\mathrm{c}}$ for $\beta_{1} \rightarrow \beta_{1}^{\prime}$ transformation increases linearly with increasing $T$, 
whereas that for $\beta_{1}^{\prime} \rightarrow \alpha_{1}^{\prime}$ transformation decreases linearly. Thus, $\sigma_{\mathrm{c}}$ vs $T$ for the two transformations forms two straight lines with the slopes of opposite signs in the phase diagram (see Fig. 9 in Ref. (2)). However, above $470 \mathrm{~K}$, they deviated downwards and upwards, respectively, from the straight lines, and an anomaly is observed that the sign of $\mathrm{d} \sigma_{\mathrm{c}} / \mathrm{d} T$ for $\beta_{1}^{\prime} \rightarrow \alpha_{1}^{\prime}$ transformation changes from negative to positive at around $470 \mathrm{~K}$. As a result, $\sigma_{\mathrm{c}}$ for $\beta_{1}^{\prime} \rightarrow \alpha_{1}^{\prime}$ transformation is always higher than that for $\beta_{1} \rightarrow \beta_{1}^{\prime}$ transformation even though $T$ is elevated further, and the lines of $\sigma_{\mathrm{c}}$ vs $T$ for the two transformations never intersect each other. The stress-strain (S-S) curves associated with those transformations show two distinct stages consisting of the lower one due to $\beta_{1} \rightarrow \beta_{1}^{\prime}$ transformation and the upper one due to $\beta_{1}^{\prime} \rightarrow \alpha_{1}^{\prime}$ transformation. These facts suggest that the stress-induced $\beta_{1}^{\prime}$ martensite may metastably exist even at elevated temperature. It is to be noted here that the $\beta_{1}$ parent phase is obtaind by quenching from a high temperature, and therefore, that all the martensite phases derived directly or successively from the $\beta_{1}$ parent are metastable by nature.

On the other hand, it has been reported ${ }^{(3)-(5)}$ in $\mathrm{Cu}-\mathrm{Zn}-\mathrm{Al}$ alloys that $\sigma_{\mathrm{c}}$ for $\beta_{1} \rightarrow \beta_{1}^{\prime}$ transformation becomes higher than that for $\beta_{1}^{\prime} \rightarrow \alpha_{1}^{\prime}$ transformation above a certain temperature which is not so high as in $\mathrm{Cu}-\mathrm{Al}-\mathrm{Ni}$ alloys. The $\beta_{1}^{\prime}$ martensite stress-induced from the $\beta_{1}$ parent phase in $\mathrm{Cu}-\mathrm{Zn}-\mathrm{Al}$ transforms immediately into $\alpha_{1}^{\prime}$ martensite by the intersection of two variants of $\beta_{1}^{\prime}$ martensites, and the associated S-S curve shows a certain amount of stress drop when $\alpha_{1}^{\prime}$ martensite is formed ${ }^{(13)}$. Such an immediate transformation of $\beta_{1}^{\prime}$ martensite to another one and the stress drop in the S-S curve suggest that the $\beta_{1}^{\prime}$ martensite is an intermediate transition phase and has no stress range where it can metastably exist.

From the comparison between $\mathrm{Cu}-\mathrm{Al}-\mathrm{Ni}$ and $\mathrm{Cu}-\mathrm{Zn}-\mathrm{Al}$ alloys, it is supposed that the anomaly in $\mathrm{d} \sigma_{\mathrm{c}} / \mathrm{d} T$ and the presence of matastable $\beta_{1}^{\prime}$ martensite at elevated temperature in the former alloy are due to some aging effect occurring during the tensile tests. If this supposition is correct, $\sigma_{\mathrm{c}}$ and other characteristics may be influenced by heat treatment. This is also expected from the fact that the phase diagram previously determined is a metastable equilibrium one, which may vary with the cooling velocity.

Many investigations have been done on the effect of heat treatments, such as quenching velocity from a high temperature region and aging treatment after quenching, on transformation characteristics in $\mathrm{Cu}$-based shape memory alloys ${ }^{(6)-(16)}$. However, only a few studies have been done on effects of heat treatments on the stress-induced transformation or the transformation pseudoelasticity ${ }^{(17)(18)}$ and on the phase diagram in the temperature-stress coordinates. The purpose of the present study is to clarify these effects along with the origin of the anomaly in $\mathrm{d} \sigma_{\mathrm{c}} / \mathrm{d} T$ for $\beta_{1}^{\prime} \rightarrow \alpha_{1}^{\prime}$ transformation, that is, how $\sigma_{c}$, stress hysteresis $H$ and transformation strain $\Delta \varepsilon$ are influenced by low temperature aging of the $\beta_{1}$ parent and by quenching velocity from high temperature $\beta$ phase has been investigated. These heat treatments are naturally considered to bring in some changes of many factors, such as domain size, degree of order, vacancy concentration, lattice constant, precipitation and so on, in the alloy structure. Actually, domain size of ordered structure has been found to vary with heat treatment ${ }^{(20)}$. Thus, the variation in transformation pseudoelasticity characteristics observed here seems to be mostly related to the domain size, although some other factors have to be also taken into consideration. In the present paper, however, description will be concentrated mainly on the variation of tranformation pseudoelasticity characteristics.

\section{Experimental Procedures}

Two alloys, alloy $1(\mathrm{Cu}-14.1 \mathrm{Al}-4.2 \mathrm{Ni}$ (mass $\%)$ ) and alloy $2(\mathrm{Cu}-14.7 \mathrm{Al}-4.2 \mathrm{Ni}(\operatorname{mass} \%)$ ), are used in the present study. Single crystals, whose orientations were within a few degrees from $\langle 001\rangle$, were grown by a modified Bridgman method. After shaping the single crystals into a suitable size (gauge length portion being $20 \mathrm{~mm} \times 4 \mathrm{~mm} \times 2 \mathrm{~mm}$ ) for tensile tests, they were subjected to the following three kinds of heat treatments:

(a) After being solution-treated at $1273 \mathrm{~K}$ 
for $300 \mathrm{~s}$, alloys 1 and 2 were quenched to 265 and $253 \mathrm{~K}$, respectively. The specimens were dropped into $10 \% \mathrm{NaOH}$ solution from a vertical furnace.

(b) After being solution-treated at $1273 \mathrm{~K}$ for $3.6 \mathrm{ks}$, alloys 1 and 2 were quenched into water at 325 and $273 \mathrm{~K}$, respectively. A horizontal type vacuum furnace was used for this treatment. The quenching velocity in the treatments (a) and (b) was not measured, but a large difference seems to surely exist between them.

(c) The alloys were aged at $523 \mathrm{~K}$ for $3.6 \mathrm{ks}$ after the treatment (b) and subsequently quenched into water at room temperature.

The above three kinds of heat treatments will be called here "rapidly quenched", " normally quenched" and "aged", respectively. All the heat treatments were done on an identical specimen to avoid a scatter of results arising from use of different specimens. That is, a specimen subjected to one kind of the heat treatments was first tensile-tested as a function of temperature, and successively the same specimen was subjected to another kind of the heat treatments. By using a TT-CM-L type Instron machine, the tensile-tests have been done at temperatures below $470 \mathrm{~K}$ where no aging effect is expected to occur, the strain rate being $4.17 \times 10^{-3} / \mathrm{s}$. In the tensile tests at temperatures below $M_{s}$, specimens with a single variant $\gamma_{1}^{\prime}$ martensite were used, which were obtained by the method described in Ref. (19). An extensometer was used between 223 and $423 \mathrm{~K}$ but not below $223 \mathrm{~K}$. Alloy 1 was extended up to the end of the second stage to measure $\Delta \varepsilon$ for $\beta_{1}^{\prime}\left(\beta_{1}^{\prime \prime}\right) \rightarrow \alpha_{1}^{\prime}$ as well as those for $\beta_{1} \rightarrow \beta_{1}^{\prime}$ and $\gamma_{1}^{\prime} \rightarrow \beta_{1}^{\prime \prime}$, unless otherwise stated. $M_{s}$ temperatures were measured by observing the surface relief on the specimens. The method of the specimen preparation was almost the same as in the previous paper ${ }^{(1)}$.

\section{Results and Discussion}

\section{1. $M_{\mathrm{s}}$ temperature and thermal hysteresis}

Figure 1 shows the variation of $\mathrm{M}_{\mathrm{s}}$ temperatures of alloys 1 and 2 with heat treatment. In most cases, the martensite formed upon cool-

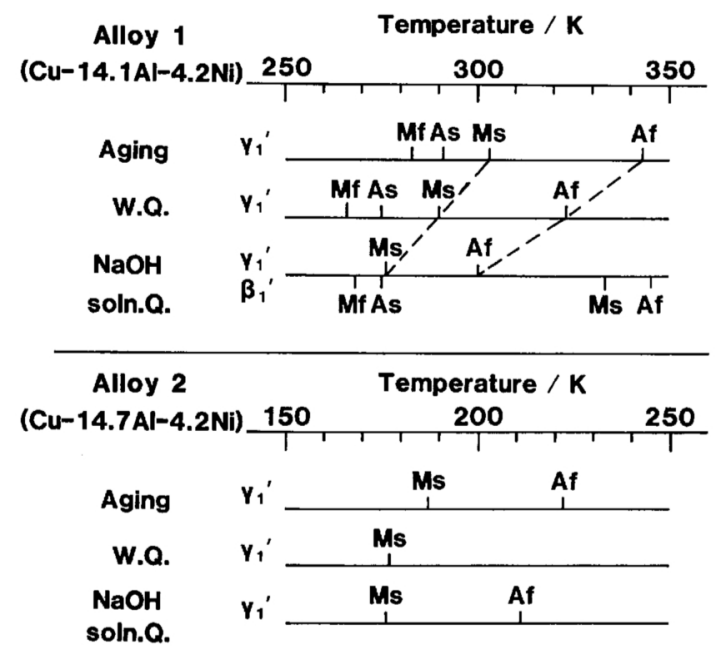

Fig. 1 Transformation temperatures of alloys 1 and 2 subjected to three kinds of heat treatments.

ing was acicular type $\gamma_{1}^{\prime} . M_{s}$ for the $\gamma_{1}^{\prime}$ rises in the order of the rapidly quenched, normally quenched and aged specimens, although the change in alloy 2 is small. The thermal hysteresis, $A_{f}-M_{s}$, also increases in the same order. The variation in this order will be called "regular order". The rise in $\mathbf{M}_{\mathrm{s}}$ and the increase in thermal hysteresis for aged specimens are in accordance with the results obtained by other investigators $^{(6)-(11)}$. In the rapidly quenched specimen of alloy 1 , banded $\beta_{1}^{\prime}$ martensite whose $M_{s}$ is higher than for the $\gamma_{1}^{\prime}$ is additionally formed. A microstructural memory effect on thermal cycling was observed in this specimen and its details will be described elsewhere ${ }^{(20)}$.

To clarify the origin for the variation in transformation characteristics, order domains in alloys 1 and 2 were observed by transmission electron microscopy. As a result, it was found that the domain size in alloy 1 became larger in the regular order but that the change in alloy 2 was very small. Besides, the degree of order in alloy 2 whose $M_{s}$ was below room temperature was measured by using an X-ray four-circle diffractometer, but no change was observed with hat treatment. From these experiments, the variation in $M_{s}$ will be ascribed largely to the variation in domain size, although there may be other factors. The variation in transformation pseudoelasticity characteristics with heat treatment describved below also seems to 
be ascribed chiefly to the change in domain size. Detailed discussion from the viewpoint of changes in alloy structures will be given elsewhere along with thermodynamical analysis ${ }^{(20)}$.

\section{Stress-stain curve}

Figures 2 and 3 show stress-strain (S-S) curves tested at various temperatures above $\mathbf{M}_{\mathrm{s}}$ for alloys 1 and 2, respectively. Alloy $1 \mathrm{ex}-$ hibits a pseudoelastic loop of type B at 323 and $373 \mathrm{~K}$ except for the aged specimen tested at $323 \mathrm{~K}$, and a pseudoelastic loop of type $\mathrm{C}$ at $423 \mathrm{~K}$. Meanwhile, alloy 2 was not deformed up to the second state, since $\sigma_{\mathrm{c}}$ for its $\beta_{1}^{\prime} \rightarrow \alpha_{1}^{\prime}$ transformation is expected to be higher than the fracture stress. Thus, pseudoelastic loops due to $\beta_{1} \rightleftarrows \beta_{1}^{\prime}$ transformations appear at 223 , 273 and $323 \mathrm{~K}$ except for the aged specimen tested at $223 \mathrm{~K}$. On the other hand, stress-induced $\beta_{1} \rightarrow \gamma_{1}^{\prime}$ transformation occurs at $193 \mathrm{~K}$ in the vicinity of $M_{s}$. This is well indicated by the $\mathrm{S}-\mathrm{S}$ characteristics, such as the existence of peaks following the elastic deformation of $\beta_{1}$ parent, subsequent somewhat serrated flow and residual strain when unloaded (no extensometer was used).

The irregular curves appearing in both the figures may be explained as follows. The one for the aged specimen of alloy 1 tested at $323 \mathrm{~K}$ (a) $\mathrm{NaOH}$ soln. quench

(i) $193 \mathrm{~K}$

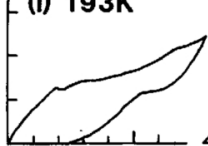

(ii) 223K
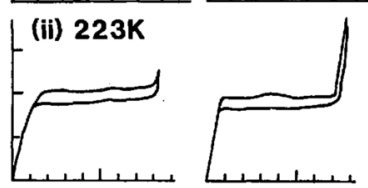

Water quench

(c) Aging at 523K for $3.6 \mathrm{ks}$

(iii) $273 \mathrm{~K}$
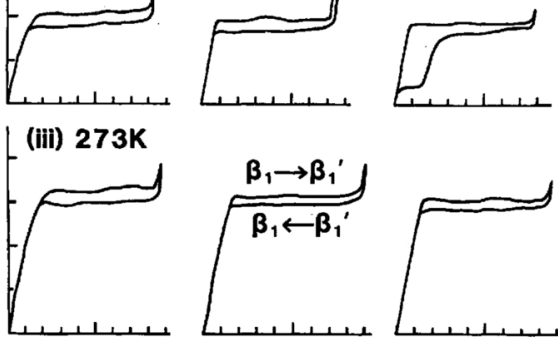

(iv) 323K

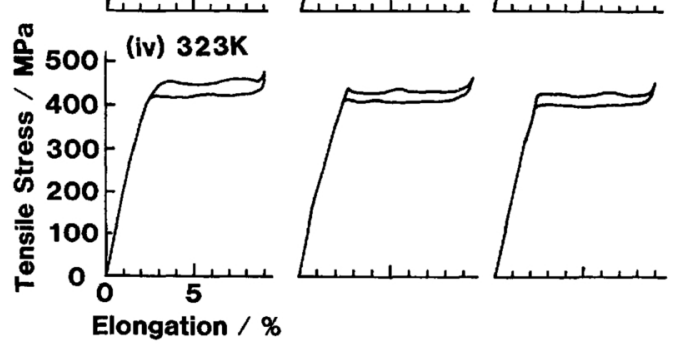

Fig. 3 Stress-strain curves as a function of temperature above $M_{s}$ for alloy 2 subjected to three kinds of heat treatments (extensometer was not used at $193 \mathrm{~K}$ ). (a) $\mathrm{NaOH}$ soln. quench

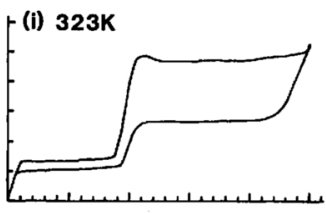

(ii) $373 K$

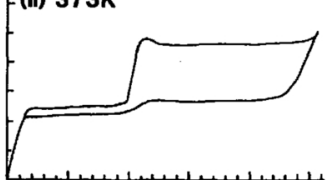

$\frac{2}{2} 600$

600 (iii) $423 K$

500

总 300

की 200

100.

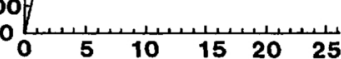

Elongation / \% (b) Water quench
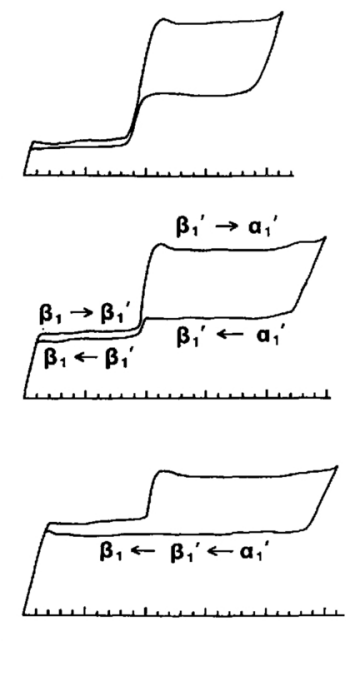

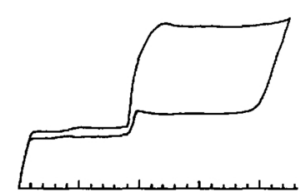

(c) Aging at $523 \mathrm{~K}$ for $3.6 \mathrm{ks}$
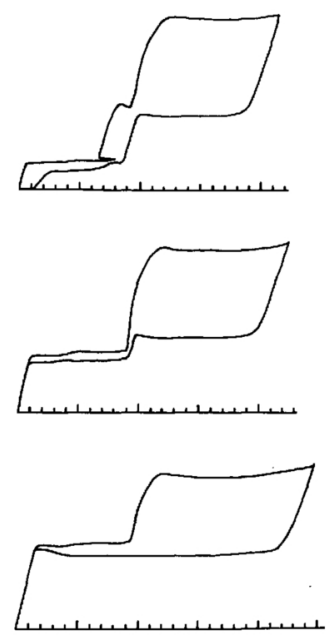

Fig. 2 Stress-strain curves as a function of temperature above $\mathbf{M}_{\mathrm{s}}$ for alloy 1 subjected to three kinds of heat treatments. 
(Fig. 2) appears possibly because the test temperature is between $M_{s}(304 \mathrm{~K})$ and $A_{f}(343 \mathrm{~K})$. The strain retrogresses at the end of the first stage, because a part of stress-induced $\beta_{1}^{\prime}$ transforms into $\gamma_{1}^{\prime}$, which subsequently transforms into $\beta_{1}^{\prime \prime}$ at the second stage. At the following third stage, a mixture of the $\beta_{1}^{\prime}$ and $\beta_{1}^{\prime \prime}$ transforms into $\alpha_{1}^{\prime}$. On unloading, the $\alpha_{1}^{\prime}$ transforms back to $\beta_{1}^{\prime}$, which subsequently transforms partly into $\beta_{1}$ and partly into $\gamma_{1}^{\prime}$. Thus, some strain due to the $\gamma_{1}^{\prime}$ remains under no external stress. On the other hand, the curve for the aged specimen of alloy 2 tested at $223 \mathrm{~K}$ (Fig. 3) appears because the stress-induced $\beta_{1}^{\prime}$ transforms into $\gamma_{1}^{\prime}$ on the halfway of unloading.

Figures 4 and 5 (no extensometer was used in the later tensile test) show the variation of S-S curves with $T$ below $\mathrm{A}_{\mathrm{f}}$ for alloys 1 and 2, respectively. Alloy 1 shows pseudoelastic loops of type A except for the aged specimen which shows no second stage on loading up to 600 $\mathrm{MPa}$. Alloy 2 was deformed so that only one stage pseudoelasticity appeared. It is to be noted here that the feature of the first stage due to the $\gamma_{1}^{\prime} \rightarrow \beta_{1}^{\prime \prime}$ transformation differs depending on the alloy composition and heat treatment. That is, in alloy 2 , the S-S curve at this stage is all serrated on both loading and unloading (a) $\mathrm{NaOH}$ soln. quench

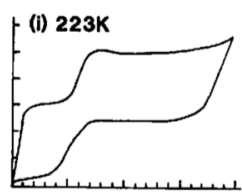

(b) Water quench
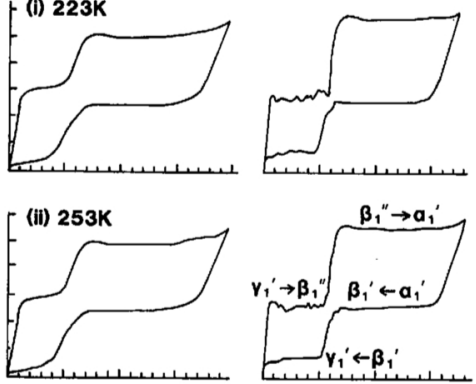

을 600 (iii) $283 \mathrm{~K}$
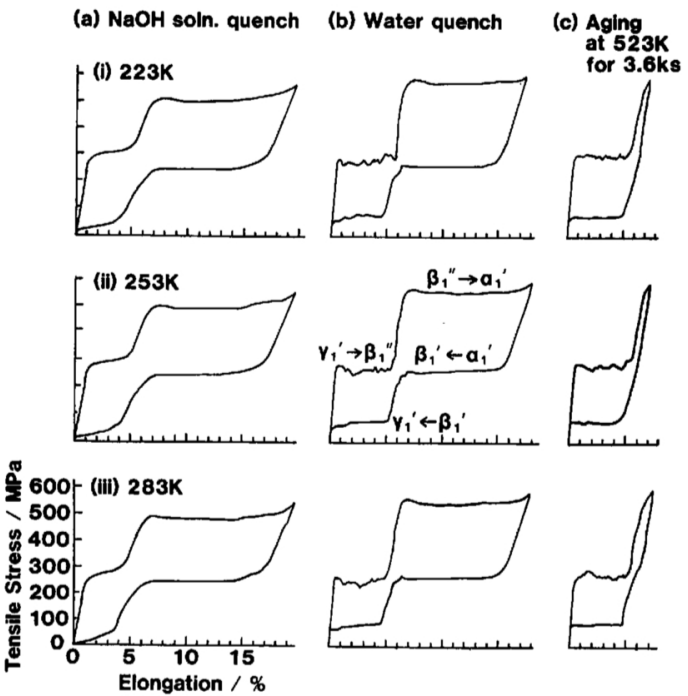

Fig. 4 Stress-strain curves as a function of temperature below $A_{f}$ for alloy 1 subjected to three kinds of heat treatments. (a) $\mathrm{NaOH}$ soln. quench
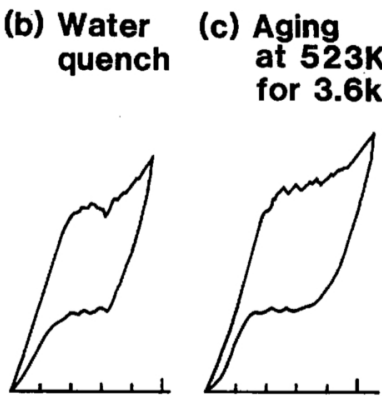

(ii) $163 \mathrm{~K}$
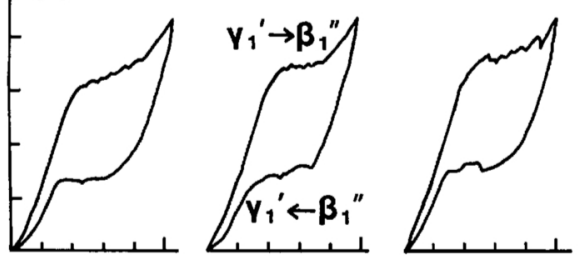

里
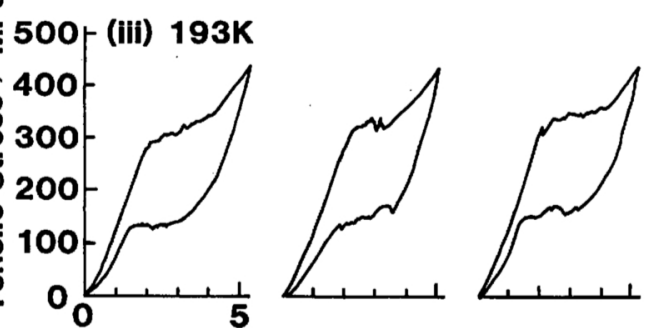

(c) Aging at $523 K$ for 3.6ks

Elongation / \%

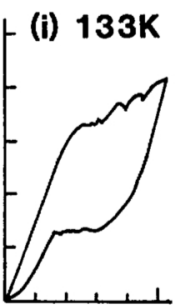

Fig. 5 Stress-strain curves as a function of temperature below $A_{f}$ for alloys 2 subjected to three kinds of heat treatments (no extensometer was used).

regardless of the heat treatment. In alloy 1 , on the other hand, the S-S curve on loading for the rapidly quenched specimen is smooth and gently slant, whereas those for the other specimens are serrated but almost flat. These differences are due probably to different transformation modes of $\gamma_{1}^{\prime} \rightarrow \beta_{1}^{\prime \prime}$. This will be described in detail elsewhere ${ }^{(20)}$.

Further, by closely examining these S-S curves, $\Delta \varepsilon$ on loading $\left(\Delta \varepsilon^{L}\right)$ is seen to slightly differ from that on unloading $\left(\Delta \varepsilon^{\mathrm{U}}\right)$ in various tranformations. Besides, some deviation from a linear relation can be seen, more or less, between stress and strain in the elastic region of various phases on the curves (the elastic region of $\alpha_{1}^{\prime}$ phase appears on unloading). The existence of such a non-linear elasticity, which has been considered to be due to the lattice anharmonicity of crystals ${ }^{(21)-(23)}$, indicates that 
higher order elastic constants play a significant role in the elastic deformation of various phases in the present $\mathrm{Cu}-\mathrm{Al}-\mathrm{Ni}$ alloy. This non-linear elasticity was not definitely distinguished from pseudoelasticity in a previous paper $^{(24)}$.

From a comparison between S-S curves at the same test temperature for the specimens subjected to different heat treatments, it is clearly seen that $\sigma_{\mathrm{c}}$ on loading $\left(\sigma_{\mathrm{c}}^{\mathrm{L}}\right)$ and on unloading $\left(\sigma_{\mathrm{c}}^{\mathrm{U}}\right)$ and hence stress hysteresis $\left(H=\sigma_{\mathrm{c}}^{\mathrm{L}}-\sigma_{\mathrm{c}}^{\mathrm{U}}\right)$ for various transformations vary with heat treatment. $\Delta \varepsilon$ for various transformations, Young's modulus $(E)$ and elastic nonlinearity of various phases also vary with heat treatment and temperature. $\sigma_{\mathrm{c}}^{\mathrm{L}}, H$ and $\Delta \varepsilon^{\mathrm{L}}$ have been plotted as a function of $T$, setting the kind of heat treatment as a parameter. These will be described in the following sections.

\section{Critical stress}

Figures 6 and 7 show $\sigma_{\mathrm{c}}^{\mathrm{L}}$ vs $T$ relations for various transformations in alloys 1 and 2 , respectively. $\sigma_{\mathrm{c}}^{\mathrm{L}}$ clearly bears a linear relation to $T$ below $470 \mathrm{~K}$ for all the transformations (the bend seen in Fig. 7 corresponds to the transition of transformation type, that is, $\beta_{1} \rightarrow$ $\gamma_{1}^{\prime}$ to $\beta_{1} \rightarrow \beta_{1}^{\prime}$ as previously clarified $\left.{ }^{(1)}\right)$. Besides, the slopes of these straight lines are almost the same regardless of the heat treatment. Therefore, the effect of heat treatment may be evaluated simply by comparing $\sigma_{c}^{\mathrm{L}}$ at a certain temperature.

The variation of $\sigma_{\mathrm{c}}^{\mathrm{L}}$ with heat treatment is as follows. $\sigma_{\mathrm{c}}^{\mathrm{L}}$ for almost all types of transforma-

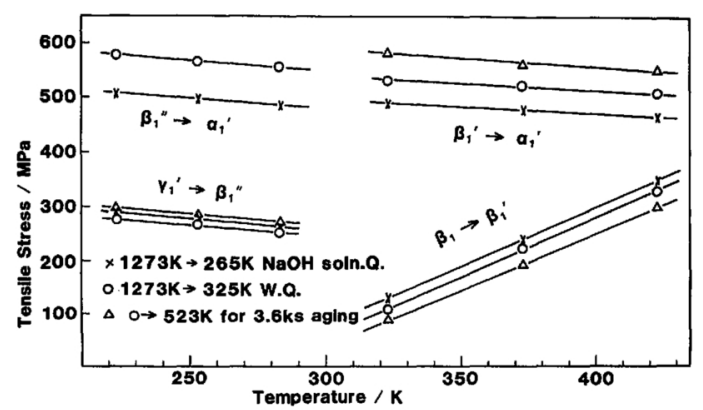

Fig. 6 Effect of heat treatments on critical stresses indicated as a function of temperature for each transformation in alloy 1 .

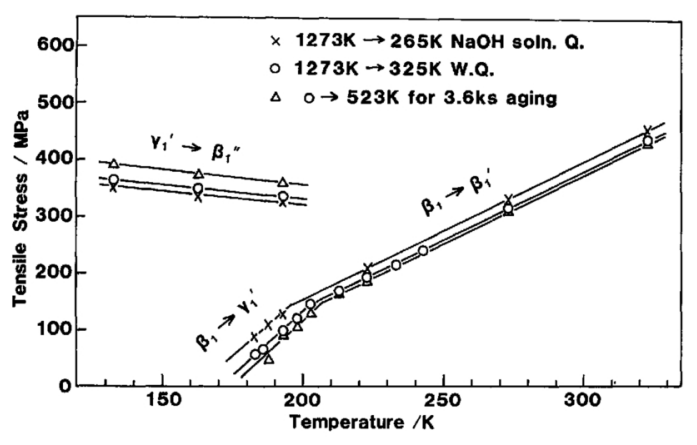

Fig. 7 Effect of heat treatments on critical stresses indicated as a function of temperature for each transformation in alloy 2 .

tions in both the alloys decreases or increases in the "regular order" (this will be termed a "downward" or "upward" variation), except for the $\gamma_{1}^{\prime} \rightarrow \beta_{1}^{\prime \prime}$ transformation in alloy 1 in which $\sigma_{c}^{\mathrm{L}}$ increases in the order of the normally quenched, rapidly quenched and aged specimens. The "downward" variation of $\sigma_{\mathrm{c}}^{\mathrm{L}}$ for $\beta_{1} \rightarrow \gamma_{1}^{\prime}$ transformation in alloy 2 is consistent with the corresponding rise in $\mathbf{M}_{\mathrm{s}}$ temperature in the "regular order". The reason for the different variation of $\sigma_{c}^{\mathrm{L}}$ in $\gamma_{1}^{\prime} \rightarrow \beta_{1}^{\prime \prime}$ in alloy 1 seems to be due to a variation of the transformation mode with heat treatment, as noted in the previous section. Accordingly, it may be generally said that $\sigma_{\mathrm{c}}^{\mathrm{L}}$ for the transformations from parent to martensites exhibits the "downward" variation whereas $\sigma_{c}^{\mathrm{L}}$ for the transformation between martensites shows the "upward" variation. This tendency is in coincidence with the downward and upward deviations of the $\sigma_{c}$ vs $T$ relation for the two transformations above $470 \mathrm{~K}$ from the linear relationship observed in the previous study ${ }^{(2)}$. This means that the previously reported anomalous deviation in the $\sigma_{\mathrm{c}}$ vs $T$ relation is due to an aging effect occurring during tensile tests, not due to the absence of mechanism for the direct $\beta_{1} \rightarrow \alpha_{1}^{\prime}$ transformation. Another evidence for this is that the occurrence of consecutive $\beta_{1} \rightleftarrows \beta_{1}^{\prime} \rightleftarrows \alpha_{1}^{\prime}$ transformation pseudoelasticity has been found in alloys with slightly different composition below $470 \mathrm{~K}^{(20)}$, as in $\mathrm{Cu}-\mathrm{Zn}-\mathrm{Al}$ alloys ${ }^{(3)-(5)}$.

$\sigma_{\mathrm{c}}^{\mathrm{U}}$ is also seen to bear a linear relation to $T$. However, the variation of $\sigma_{\mathrm{c}}^{\mathrm{U}}$ with heat treatment is found to be rather complicated, since 
$H\left(=\sigma_{\mathrm{c}}^{\mathrm{L}}-\sigma_{\mathrm{c}}^{\mathrm{U}}\right)$ also varies with heat treatment, as will be described in the next section.

As described above, both $\sigma_{\mathrm{c}}^{\mathrm{L}}$ and $\sigma_{\mathrm{c}}^{\mathrm{U}}$ vary with heat treatment, and therefore, the equilibrium stress $\left(\sigma^{\mathrm{E}}\right)$ which is the mid-point between $\sigma_{\mathrm{c}}^{\mathrm{L}}$ and $\sigma_{\mathrm{c}}^{\mathrm{U}}$, also vary with heat treatment. Hence, we can say that the metastable equilibrium phase diagram of $\mathrm{Cu}-\mathrm{Al}-\mathrm{Ni}$ alloys in the temperature-stress coordinates varies with heat treatment, as expected.

\section{Stress hysteresis}

According to the Sumino and Suezawa's theory ${ }^{(25)}$, wherein a surface dislocation theory for deformation twinning ${ }^{(26)}$ was expanded to a stress-induced martensitic transformation, $H$ should show a linear relation to the reciprocal of temperature $(1 / T)$ with a positive slope. In fact, a linear relation between $H$ and $1 / T$ has recently been found for the $\gamma_{2}^{\prime} \rightleftarrows \beta_{2}^{\prime \prime}$ transformations in $\mathrm{Au}-47.5$ at $\% \mathrm{Cd}$ alloy single crystals ${ }^{(27)}$. $H$ for various transformations in $\mathrm{Cu}-\mathrm{Al}-\mathrm{Ni}$ alloys was previously explained in terms of this theory by Otsuka et al. ${ }^{(1)}$. However, this theory is not applicable to the present $\mathrm{Cu}-\mathrm{Al}-\mathrm{Ni}$ alloys. The reason is that, since both $\sigma_{\mathrm{c}}^{\mathrm{L}}$ and $\sigma_{\mathrm{c}}^{\mathrm{U}}$ show a linear relation to $T$ according to the Clausius-Clapeyron equation, $H$ must show also a linear relation to $T$, but not $1 / T$.

Figure 8 shows the $H$ vs $T$ relation for alloys 1 and 2. With increasing $T, H$ for the $\gamma_{1}^{\prime} \rightleftarrows \beta_{1}^{\prime \prime}\left(\beta_{1}^{\prime}\right)$ and $\beta_{1}^{\prime}\left(\beta_{1}^{\prime \prime}\right) \rightleftarrows \alpha_{1}^{\prime}$ transformations decreases, whereas that for the $\beta_{1} \rightleftarrows \beta_{1}^{\prime}$ transformations slighly increases. These relations are qualitatively in accordance with those in the previous study ${ }^{(1)}$.

The variation of $H$ with heat treatment is similar to that of $\sigma_{c}^{\mathrm{L}}$ for a certain type of transformation. For example, $H$ for $\beta_{1}^{\prime} \rightleftarrows$ $\left(\beta_{1}^{\prime \prime}\right) \rightleftarrows \alpha_{1}^{\prime}$ transformations increases in the regular order, and those for $\beta_{1} \rightleftarrows \beta_{1}^{\prime}$ transformations appear to decreases in the regular order, although $H$ values of the aged and normally quenched specimens are almost the same. However, for $\gamma_{1}^{\prime} \rightleftarrows \beta_{1}^{\prime \prime}\left(\beta_{1}^{\prime}\right)$ transformations, the variation of $H$ with heat treatment is different from that of $\sigma_{\mathrm{c}}^{\mathrm{L}}$, i.e., the $H$ in alloy 1 increases in nearly the regular order but that in alloy 2 decreases in the order of the normally quenched, rapidly quenched and aged specimens. $H$

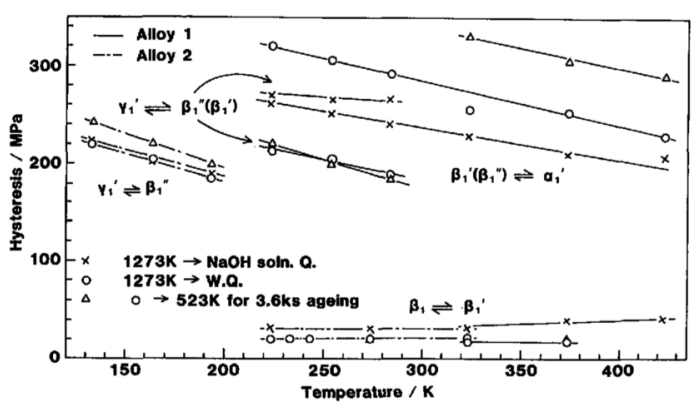

Fig. 8 Effect of heat treatments on stress hysteresis indicated as a function of temperature for each transformation in alloys 1 and 2 .

of $\beta_{1} \rightleftarrows \gamma_{1}^{\prime}$ transformations was not measured, because the stress-induced $\gamma_{1}^{\prime}$ did not revert on unloading. However, it appears to increase in the regular order as that for $\beta_{1} \rightleftarrows \beta_{1}^{\prime}$ transformations, being consistent with the increase of thermal hysteresis of transformation temperature in the regular order.

\section{Transformation elongation}

Figure 9 shows $\Delta \varepsilon^{L}$ as a function of $T$ for various transformations in alloy 1 , in which $\Delta \varepsilon^{\mathrm{L}}$ values calculated from lattice constants of $\beta_{1}$ parent and various martensitic phase for the present orientation ${ }^{(1)(28)}$ are also shown. It is clearly seen that $\Delta \varepsilon^{\mathrm{L}}$ is not constant but varies not only with $T$ but also with heat treatment.

First, it is to be noted that $\Delta \varepsilon$ for $\beta_{1}^{\prime}\left(\beta_{1}^{\prime \prime}\right) \rightleftarrows \alpha_{1}^{\prime}$ transformation increases whereas that for $\gamma_{1}^{\prime} \rightarrow \beta_{1}^{\prime \prime}$ transformation decreases with increasing $T$. Such a negative temperature dependence of $\Delta \varepsilon$ has recently been observed similarly for the $\gamma_{2}^{\prime} \rightarrow \beta_{2}^{\prime \prime}$ transformation in $\mathrm{Au}-47.5$ at $\% \mathrm{Cd}$ single crystals by the present authors ${ }^{(27)}$. On the other hand, the temperature dependence of $\Delta \varepsilon$ for $\beta_{1} \rightarrow \beta_{1}^{\prime}$ transformation shows a generous convex curve. This was already reported in the previous study ${ }^{(1)}$, but the reason is not known.

Second, the variation of $\Delta \varepsilon$ with heat treatment may be characterized as follows; for $\beta_{1}^{\prime}\left(\beta_{1}^{\prime \prime}\right) \rightarrow \alpha_{1}^{\prime}$ transformation, $\Delta \varepsilon$ decreases in the regular order, and this variation is inverse to that of $\sigma_{\mathrm{c}}^{\mathrm{L}}$. Meanwhile, $\Delta \varepsilon$ for $\gamma_{1}^{\prime} \rightarrow \beta_{1}^{\prime \prime}$ transformation increases in the order of the rapidly quenched, aged and normally quenched specimens, and $\Delta \varepsilon$ for $\beta_{1} \rightarrow \beta_{1}^{\prime}$ transformation increases in the order of the aged, rapidly 


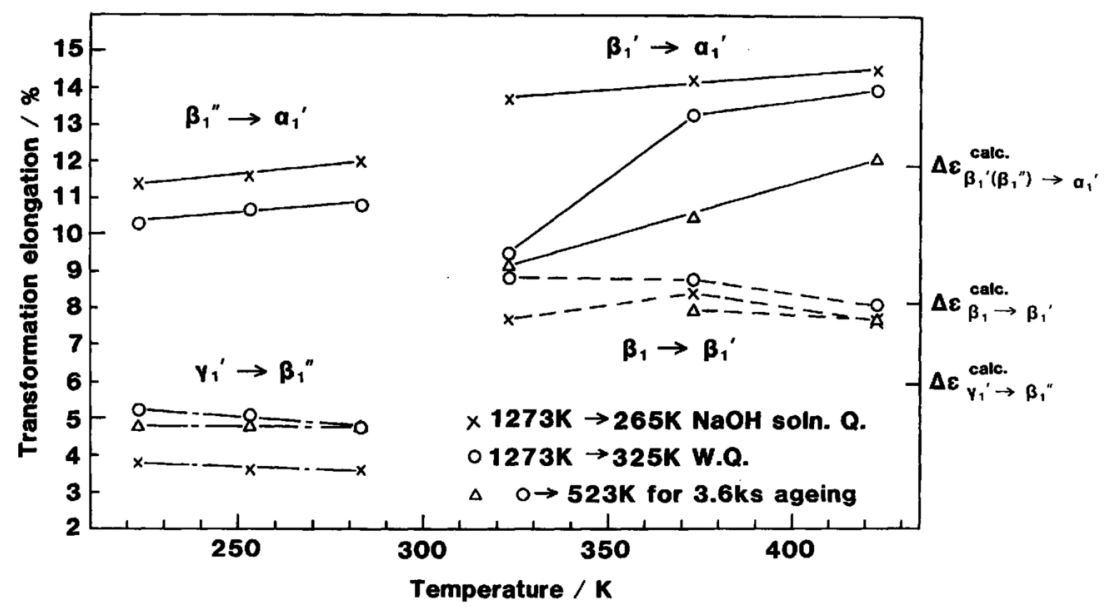

Fig. 9 Effect of heat treatments on transformation strain indicated as a function of temperature for each transformation in alloy 1 .

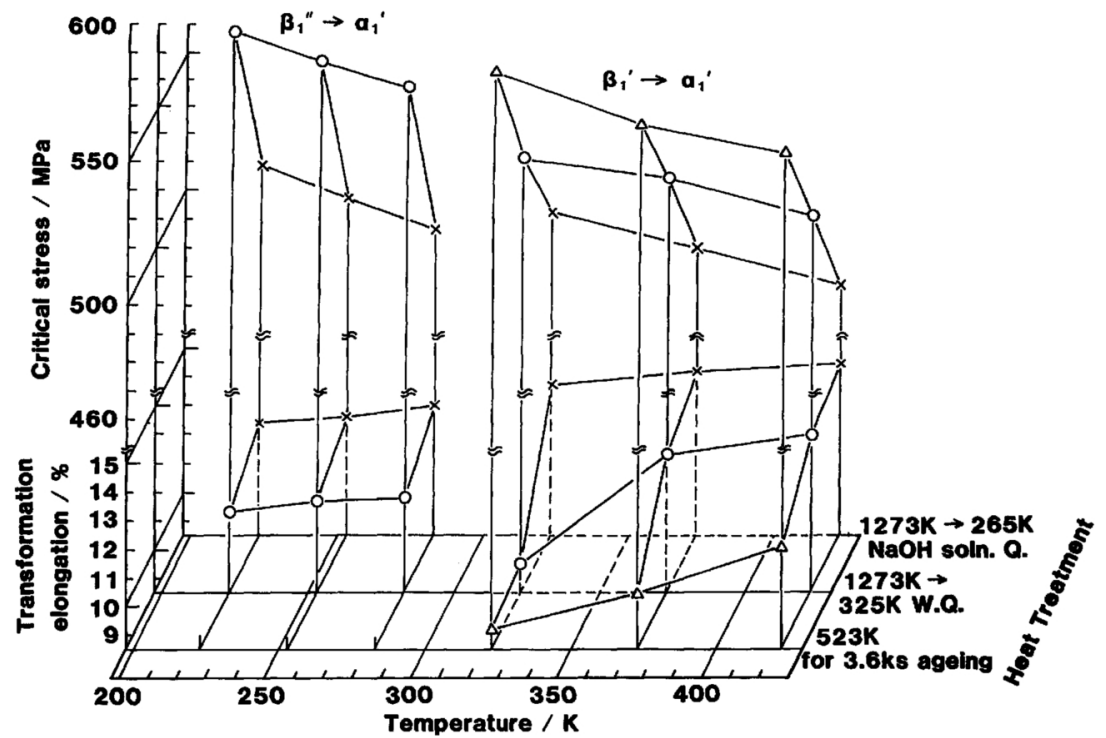

Fig. $10 \sigma_{\mathrm{c}}$ and $\Delta \varepsilon$ for $\beta_{1}^{\prime}\left(\beta_{1}^{\prime \prime}\right) \rightarrow \alpha_{1}^{\prime}$ transformation-temperature-heat treatment plots for alloy 1 .

quenched and normally quenched specimens, that is, they vary in an irregular order with heat treatment.

The above variation of $\Delta \varepsilon$ with $T$ and heat treatment seems to be ascribed to a certain variation in lattice constants of various phases, since $\Delta \varepsilon$ is derived from their lattice constants. However, for the $\beta_{1}^{\prime} \rightarrow \alpha_{1}^{\prime}$ transformation, the variation of about $20 \%$ in lattice constant is needed to explain the difference between maximum $\Delta \varepsilon(14.5 \%$ for the rapidly quenched specimen tested at $423 \mathrm{~K}$ ) and minimum $\Delta \varepsilon$ $(9.2 \%$ for the aged specimen tested at $323 \mathrm{~K})$. Although this large variation may be unlikely, an experimental measurement is under way to detect the variation of lattice constant with $T$, heat treatment and applied stress.

\section{Relation between variations of $\sigma_{c}^{L}$ and} $\Delta \varepsilon$ for $\beta_{1}^{\prime}\left(\beta_{1}^{\prime \prime}\right) \rightarrow \alpha_{1}^{\prime}$ transformation

Figure 10 shows the $\sigma_{\mathrm{c}}^{\mathrm{L}}$ and $\Delta \varepsilon$ plotted as a function of $T$ for the $\beta_{1}^{\prime}\left(\beta_{1}^{\prime \prime}\right) \rightarrow \alpha_{1}^{\prime}$ transforma- 
tion in alloy 1 . It is seen from the figure that $\sigma_{\mathrm{c}}^{\mathrm{L}}$ decreases with increasing $T$ or with changing heat treatment, while $\Delta \varepsilon$ increases. A similar phenomenon, though it is inverse in sense, has been observed in a hydrostatic pressureinduced transition between $\gamma$-fcc and $\alpha$-fcc in cerium by Beecroft and Swenson ${ }^{(29)}$. They found that, with increasing $T$, a critical hydrostatic pressure for inducing the transition increases, while the volume change during the transition decreases, and suggested the existence of a critical point for the transition in cerium. In analogy with this, it may be also supposed that the equilibrium line for $\beta_{1}^{\prime}\left(\beta_{1}^{\prime \prime}\right) \rightarrow \alpha_{1}^{\prime}$ transformation in $\mathrm{Cu}-\mathrm{Al}-\mathrm{Ni}$ alloys terminates at a critical point of low temperature. This appears to be probable, since both the $\alpha_{1}^{\prime}$ and $\beta_{1}^{\prime}\left(\beta_{1}^{\prime \prime}\right)$ phases have similar structures with a long period stacking order having a common basal plane. Detailed analysis on the existence of a critical point will be described elsewhere ${ }^{(20)}$.

\section{Acknowledgements}

The present study was partly supported by the Research Project of the ISIR, Osaka University, on "Development of New Materials for Energy Conversion", and by the Grant-inAid for Fundamental Scientific Research (Ippan B, 1985-86, and Ippan C, 1986) from the Ministry of Education, Science and Culture, Japan, and the support is greatly appreciated.

\section{REFERENCES}

(1) K. Otsuka, H. Sakamoto and K. Shimizu: Acta Met., 27 (1979), 585.

(2) H. Sakamoto, K. Shimizu and K. Otsuka: Trans. JIM, 26 (1985), 638.

(3) H. Sato, K. Takezawa and S. Sato: Trans. JIM, 25 (1984), 324.

(4) H. Sato, K. Takezawa and S. Sato: ibid., 25 (1984), 332.

(5) T. Saburi, Y. Inada, S. Nenno and N. Hori: Proc. ICOMAT-82, Leuven, J. de Phys., 43 (1982), Suppl. No. 12, p. C4-633.

(6) T. Kajitani and K. Hirano: Proc. JIMIS-I
(ICOMAT-76), (1976), Kobe, Suppl. to Trans. JIM, 17 (1976), p. 117.

(7) D. P. Dunne and N. F. Kennon: Metals Forum, 4 (1981), 176.

(8) N. F. Kennon, D. P. Dunne and L. Middleton: Met. Trans., 13A (1982), 551.

(9) K. Sugimoto, T. Okamoto and K. Ohta: Collected Abstracts of the 1979 Autumn Meeting of JIM, p. 70 (in Japanese).

(10) F. Nakamura, J. Kusui, Y. Shimizu and J. Takamura: J. JIM, 44 (1980), 1302 (in Japanese).

(11) D. Hull and R. D. Garwood: J. Inst. Metals, 86 (1957-58), 485.

(12) D. Schofield and A. P. Miodownik: Metals Technology, April (1980), p. 167.

(13) N. Kuwano and C. M. Wayman: Met. Trans., 15A (1984), 621.

(14) J. M. Cook and L. M. Brown: Scripta Met., 12 (1978), 949.

(15) K. Matsushita, T. Okamoto and T. Okamoto: J. Mat. Sci., 20 (1985), 689.

(16) G. Ya Yaroslavskiy, S. Yu, Kondratev, Yu. N. Koval, R. Ya. Musyenko and B. S. Chaykovskiy: Phys. Met. Metallov., 54 (1982), 166.

(17) R. Rapacioli, M. Chandrasekaran and L. Delaey: Shape Memory Effects in Alloys (ed. J. Perkins), (1975), p. 365.

(18) R. Rapacioli and M. Chandrasekaran: Proc. ICOMAT-79, Cambridge, (1979), p. 596.

(19) K. Otsuka, H. Sakamoto and K. Shimizu: Scripta Met., 9 (1975), 491.

(20) H. Sakamoto, Y. Nakai and K. Shimizu: in preparation.

(21) M. W. Riley and M. J. Skove: Phys. Rev. B, 8 (1973), 466.

(22) H. Kobayashi and Y. Hiki: Phys. Rev. B, 7 (1973), 594.

(23) B. E. Powell and M. J. Skove: Phys. Rev., 174 (1968), 977.

(24) K. Otsuka and K. Shimizu: Metals Forum, 4 (1981), 142.

(25) K. Sumino and M. Suezawa: unpublished work (1976), cited in Ref. (1).

(26) K. Sumino: Acta Met., 14 (1966), 1607.

(27) H. Sakamoto and K. Shimizu: Scripta Met., 20 (1986), 1349.

(28) K. Otsuka, C. M. Wayman, K. Nakai, H. Sakamoto and K. Shimizu: Acta Met., 24 (1976), 207.

(29) R. I. Beecroft and C. A. Swenson: J. Phys. Chem. Solids, 15 (1960), 234. 